\title{
THE IRANIAN NATIONAL GEODATA REVISION STRATEGY AND REALIZATION BASED ON GEODATABASE
}

\author{
M.Haeri* , A.Fasihi, SM.Ayazi
}

National Cartographic Center of Iran (Khorasan Branch), 61 Vakil abad Street, Vakil abad Blvd., P.O. Box 917751619, Mashhad, Iran - morihaeri@gmail.com, fasihi.ali@gmail.com,sma@ayazi.ir

Commission IV, WG VIII

KEY WORDS: GIS, Geodatabase, revision, spatial data

\begin{abstract}
:
In recent years, using of spatial database for storing and managing spatial data has become a hot topic in the field of GIS. Accordingly National Cartographic Center of Iran (NCC) produces - from time to time - some spatial data which is usually included in some databases. One of the NCC major projects was designing National Topographic Database (NTDB). NCC decided to create National Topographic Database of the entire country-based on 1:25000 coverage maps. The standard of NTDB was published in 1994 and its database was created at the same time. In NTDB geometric data was stored in MicroStation design format (DGN) which each feature has a link to its attribute data (stored in Microsoft Access file). Also NTDB file was produced in a sheet-wise mode and then stored in a file-based style. Besides map compilation, revision of existing maps has already been started. Key problems of NCC are revision strategy, NTDB file-based style storage and operator challenges (NCC operators are almost preferred to edit and revise geometry data in CAD environments). A GeoDatabase solution for national Geodata, based on NTDB map files and operators' revision preferences, is introduced and released herein. The proposed solution extends the traditional methods to have a seamless spatial database which it can be revised in CAD and GIS environment, simultaneously. The proposed system is the common data framework to create a central data repository for spatial data storage and management.
\end{abstract}

\section{INTRODUCTION}

In recent year population growth increases and changes in building and constructions make some change on maps. Determining of these changes on the old maps requires a comprehensive strategy. In our product line for collection of base maps in 1:25000 scales we attached element attribute. These maps are called NTDB maps (National Topographic Database). These data converted to a GeoDatabase that we called NGDB (National GeoDatabase). In our strategy we use ArcSDE and each editor connect to this database with a specific version. After editing, changes could be extracted and new attribute should attach to them. This strategy can reduce data redundancy and control of data entry could apply perfectly.

\section{BACKGROUND}

\subsection{National Topographic Database (NTDB)}

NTDB refers to standard map generation and attribute data that maintain compact and fully detailed maps. In this maps attribute data and spatial data are linked together. There is sufficient predefined layer for describing each feature in the map. Spatial data have an individual geometric attribute in the map and attributes are linked to these features via a Database Management System (DBMS). This kind of maps produced due to lack of desirable and applicable GIS environment in the past.

\footnotetext{
${ }^{*}$ Corresponding author
}

NTDB provide an excellent data source for further GIS projects.

\subsection{National GeoDatabase (NGDB)}

NTDB data are not in an acceptable format for GIS projects. It should be optimized with newest format of GIS data. First of all a comprehensive data model introduced and a suitable schema for entering previous data based on predefined standards were published. This was called NGDB. In the second step all of features from NTDB must transfer to that well-defined schema. Because NTDB are stored in sheets, all of features in the boundary of sheet separated between two sheets. It makes some problem for further works or it causes difficulties for its representation. An application was established to remove this obstacle in these maps to made seamless NGDB.

\section{CONCEPTUAL FRAMEWORK OF THE PROPOSED REVISION STRATEGY FOR NGDB}

Our strategy is divided to three main parts. In each part we consider important details of revision trend. First of all, we must prepare spatial and conceptual data in a desire format. In the second step operators should extract changes and field surveying should prepare update conceptual information. In this part we prepare a user-friendly application to make this trend easier for operators. The last procedure is about controlling of 
changing that was happened. Flowchart of this procedure was shown in Figure 1.

Defining the best data model could distinguish appropriate way for strong and stable revision strategy. The optimized spatial data in our country is NTDB map that was described earlier. These data should be covert to robust data model. It should be precise and element links should enter correctly. This procedure takes some more time. Another data resource for our aim is satellite image and related control points for referencing them. Images should bring to an accurate triangulation process and become ready for detection. These two main sources of data will be used for revision procedure.

Operators play a main role in revision part. They must detect and recognize each difference between map and image. This procedure could be time consuming and operators cannot cope with very complicated rules. On the other hand the unity of data should be concerned. In our trend all of data such as spatial and conceptual must not be separated and topologic relations are an important part. For this reason ArcSDE environment for data storing was selected. Changes will be stored in a version and source of data could be in access until the whole process of updating complete.

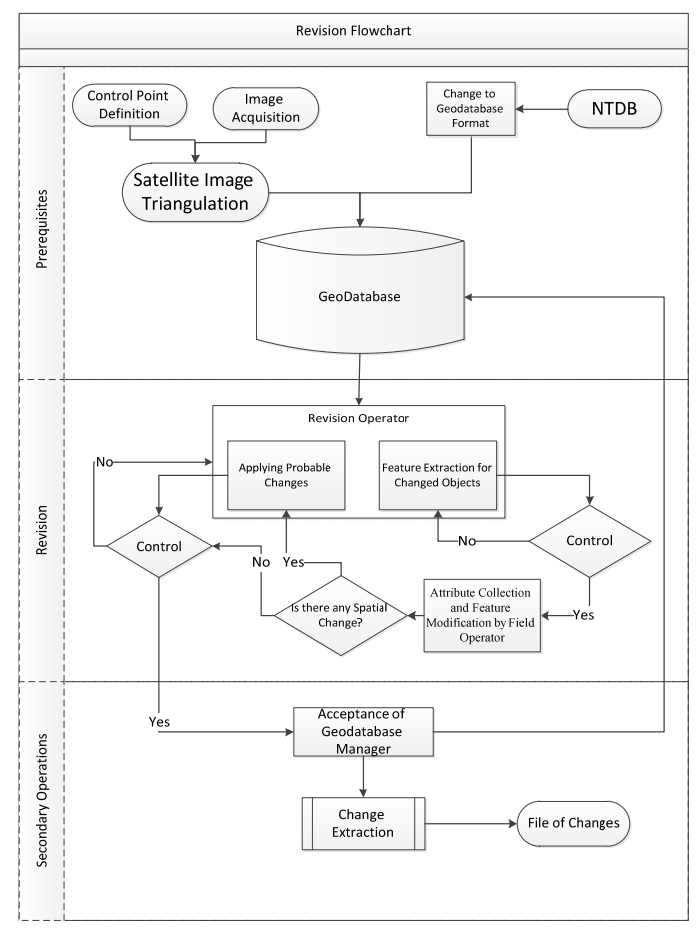

Figure 1. Revision Flowchart

This type of data storage makes some facilities for controlling updating procedure. Manager can find any differences between old generation of data and new generation. Changes could be stored in a separate file and reported.

\section{DESIGN AND IMPLEMENTATION OF A PROTOTYPE SYSTEM}

Reducing redundant data is a key feature of our strategy. Each edit will be applied to a specified version in ArcSDE. Operators choose desired sheet name and load all features in specific region. Because editing in Bentley Microstation environment is more desirable than ArcMap environment, an mdl application was established to connect with Geodatabse and editor apply changes in Microstation. Before loading data, some topology rules should be introduced. After that each data without any overlap and with regards of data interpretation priority will be loaded. An operator could edit some parts with editing tools. Links to conceptual data remain with spatial data.

Each difference extracted from satellite image in each region. Software operator could distinguish changes in building, roads, cultivations, etc. The type of building or road or another feature cannot realize from images, therefore attributes should attach by field surveying. In this step a change map will produce and an operator of field surveying search for attributes of changed objects. If any geometry modification is required it rejects to editor and applies to spatial data.

After all process on map finished, the manager of geodatabase check and control the specific version that was updated. If all changes are accepted the version post and reconcile to base version and update of that region will be finished.

\section{CONCLUSION AND FUTURE TRENDS}

The main advantage of this trend is reducing of redundant data. In the traditional method a separated file produced in each stage. By linking to a unique database all of updates could be seen by another user that is connected to it. It could be a good base for other strategies for updating by each organization which has critical connection with newest map.

This trend could be expanded to a comprehensive method for updating large amount of data by well-known CAD softwares and their tools.

\section{REFERENCES}

National Cartographic Centre of Iran, 2002. Iran Comprehensive Plan for Production of Required Maps and Spatial Information, Volume 1, National Cartographic Centre of Iran

National Cartographic Centre of Iran, 2012. Web site of National Geographic Organization of Iran, http://www.ngoiran.ir

A. Eslami Rad, M. Sarpoulaki, 2004, Islamic Republic Of Iran National Report For Photogrammetry And Remote Sensing 2000-2004, Presented to the 20th Congress of International Society for Photogrammetry and Remote Sensing. 